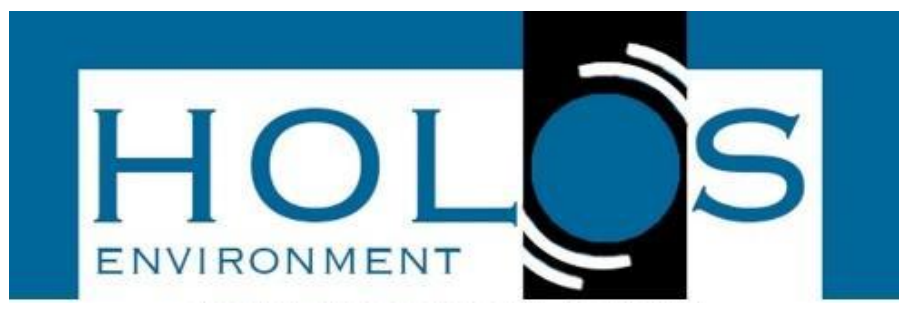

\title{
DETECÇÃO E DELIMITAÇÃO DE PEQUENOS RESERVATÓRIOS NA BACIA HIDROGRÁFICA DO RIO CACHOEIRA, BAHIA, UTILIZANDO IMAGENS MULTIESPECTRAIS
}

\section{DETECTION AND DELIMITATION OF SMALL RESERVOIRS IN THE CACHOEIRA RIVER WATERSHED, BAHIA, USING MULTISPECTRAL IMAGES}

\author{
Sabrina Brandão Cardoso ${ }^{1}$; Caroline Favoreto da Cunha ${ }^{1}$;Bruno Zanon Engelbrecht ${ }^{1}$; Hung \\ Kiang Chang ${ }^{1,2}$
}

Artigo recebido em: 20/04/2020 e aceito para publicação em: 04/07/2020. DOI: http:/dx.doi.org/10.14295/holos.v20i4.12391

\begin{abstract}
Resumo: No presente trabalho foram utilizadas imagens multiespectrais do satélite Sentinel-2 da Bacia Hidrográfica do Rio Cachoeira (BHRC), localizada no sul do estado da Bahia. O objetivo deste trabalho foi detectar, delimitar e quantificar a área ocupada por reservatórios de água na BHRC. Para tanto foram calculados os índices MNDWI (Modified Normalized Difference Water Index) e NDWI (Normalized Difference Water Index). A capacidade de detecção de pequenos corpos d'água pelos métodos empregados mostrou-se satisfatória, apresentando uma correspondência de até $78 \%$ entre os métodos, com superiores resultados para índice MNDWI frente ao NDWI. A partir desses índices foram observadas variações sazonais e espaciais quanto à distribuição de reservatórios na BHRC. A porção sudoeste da bacia apresentou maior concentração de pequenos reservatórios no período chuvoso. No contexto geral da bacia hidrográfica, os reservatórios de água ocupam até $0,13 \%$ da área da bacia, enquanto que em determinadas áreas do sudoeste da BHRC esse valor atinge até 0,86\%.
\end{abstract}

Palavras-chave: MNDWI. Bacia Hidrográfica do Rio Cachoeira. Detecção de corpos d'água.

\begin{abstract}
This work used multispectral images from the Sentinel-2 satellite of the Cachoeira River Watershed, in the south of Bahia state. This paper aim to detect, delimit and quantify the area occupied by water reservoirs at Cachoeira River Watershed. For this purpose, the MNDWI (Water Index with Modified Normalized Difference) and NDWI (Water Index with Normalized Difference) indexes were calculated. The detection capability of small water bodies by the methods employed proved to be satisfactory, with a correspondence of up to $78 \%$ between the methods, with superior results for the MNDWI index compared to the NDWI. Through this methods a seasonal and spatial changes were observed in the distribution of reservoirs in the Cachoeira River Watershed. The southwestern portion of the basin showed a higher concentration of small reservoirs in the rainy season. In the general context of the hydrographic basin, water reservoirs occupy up to $0.13 \%$ of the basin area, while in the southwestern areas of Cachoeira River Watershed this value reaches up to $0.86 \%$ of the area occupied by reservoirs.
\end{abstract}

Keywords: MNDWI. Cachoeira River Watershed. Water bodies detection.

\footnotetext{
${ }^{1}$ Universidade Estadual Paulista Júlio de Mesquita Filho (UNESP), Campus de Rio Claro, SP. E-mails: (sb.cardoso@unesp.br, caroline.favoreto@unesp.br, bruno.engelbrecht@unesp.br, chang.hung-kiang@unesp.br)

2 Laboratório de Estudo de Bacias, Unesp, Campus de Rio Claro, SP.
} 


\section{INTRODUÇÃO}

Com o crescimento da população mundial, a demanda global de água para a produção de alimentos tem experimentado forte incremento, gerando pressões sobre os recursos hídricos e aumentando sua escassez (WISSER et al., 2010).

No Brasil, a região Nordeste é intensamente afetada pela seca, possuindo drenagens incapazes de manter um fluxo perene ao longo de toda a estação do ano. Cerca de $61,7 \%$ do território nordestino encontra-se no semiárido, área que no século XIX e XX passou por sucessivas crises hídricas, ascendendo a prática da açudagem como uma alternativa para suprir a necessidade de água durante períodos de déficit hídrico. Essa prática permitiu armazenar os recursos hídricos e prolongar a estação de plantio nas áreas rurais, resultando em um aumento na quantidade de pequenos reservatórios privados ao longo dos anos.

Em razão da vasta quantidade, dimensões reduzidas e dispersão geográfica, o monitoramento hidrológico desses reservatórios requer investimentos incompatíveis com sua importância regional e com os recursos financeiros disponíveis (LIEBE et al., 2005; OGILVIE et al., 2018, 2019). Por consequência, pouca ou nenhuma informação está disponível acerca dos processos hidrológicos atuantes e do volume de água armazenado nestes reservatórios (OGILVIE et al., 2016).

Diante disso, o sensoriamento remoto apresenta-se como uma técnica eficiente para delimitação de corpos hídricos superficiais, como demonstrado por diversos trabalhos (FRAZIER et al., 2000; MUSTER et al., 2013; KAPLAN \& AVDAN, 2017; ZOU et al., 2017; CUNHA et al., 2020). No entanto, a potencialidade do sensoriamento remoto para detecção de corpos d'água com dimensões muito reduzidas é incerta e desafiam os estudos a transpor a limitação da resolução espacial dos satélites (AVISSE et al., 2017; YANG et al., 2017; ARVOR et al., 2018; OLGIVIE et al., 2018; PÔSSA e MAILLARD, 2018; DU et al. 2006). Dentre os principais satélites, o Sentinel2 possui um elevado potencial para delimitação de pequenos reservatórios (DRUSCH et al., 2012), em razão de sua resolução espacial, que varia entre $10 \mathrm{~m}$ e $60 \mathrm{~m}$, e curto período de revisita, entre 5 e 10 dias.

$O$ presente trabalho busca adensar os estudos realizados sobre a Bacia Hidrográfica do Rio Cachoeira (BHRC), uma vez que a escassez de estudos dificulta a obtenção de subsídios técnicos para a gestão dos recursos hídricos superficiais e 
subterrâneos. Dessa maneira, esse estudo pretende identificar e quantificar a área ocupada por pequenos reservatórios da Bacia Hidrográfica do Rio Cachoeira (BHRC) a partir do cálculo dos índices NDWI e MNDWI em imagens multiespectrais do satélite Sentinel-2.

\section{MATERIAIS E MÉTODOS}

\section{1 Área de estudo}

A área de estudo, com cerca de $3.953 \mathrm{~km}^{2}$, contempla a quase totalidade da Bacia Hidrográfica do Rio Cachoeira (Figura 1), situada na porção sul do estado da Bahia. A BHRC está limitada a norte pelas bacias dos rios Contas e Almada, a sul pelas bacias dos rios Pardo e Uma, a oeste pela bacia do Rio Pardo e a leste pelo Oceano Atlântico (ROCHA FILHO, 1976), abrangendo 10 municípios e uma população aproximada de 600.000 habitantes.

Figura 1 - Limites da Bacia do Rio Cachoeira, onde se insere a área de estudo

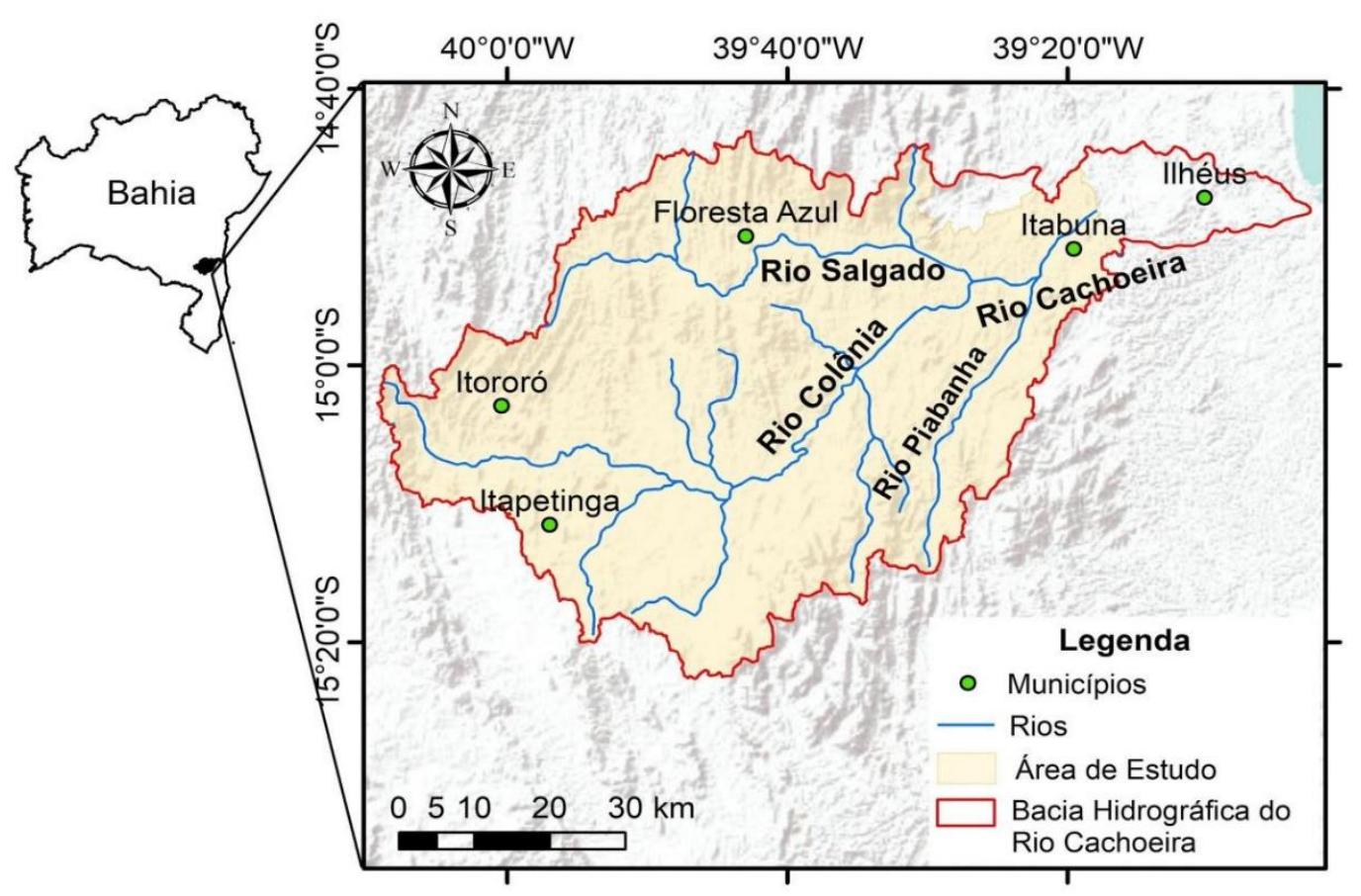




\subsubsection{Aspectos fisiográficos locais}

A BHRC está localizada na borda sudeste do Cráton São Francisco, sobre o domínio do Cinturão Itabuna-Salvador-Curaçá, formado por rochas arqueanas e paleoproterozóicas estabilizadas após o ciclo Transamazônico. De modo geral, essa unidade é formada por corpos granulitos tonalíticos e trondhjemíticos, subdivididos em séries toleíticas, cálcio-alcalinas de baixo potássio e shoshoníticas, associadas a bandas metassedimentares, além de basaltos e gabros (BARBOSA e SABATÉ, 2002). Destaca-se a presença de granulitos do Complexo Ibicaraí na porção centro-leste, e ortognaisses e ortognaissesmiloníticos do Complexo Ibicuí-Ipiaú na porção oeste. De origem pós-tectônica ocorrem corpos alcalinos de sienito, nefelina-sienito, sodalitasienito, monzodiorito, leucogabro, diorito e norito da Suíte Intrusiva Itabuna (Figura 2) (ARCANJO, 1993).

Figura 2 - Mapa geológico da área de estudo que se encontra no CinturãoSalvador-Curaçá

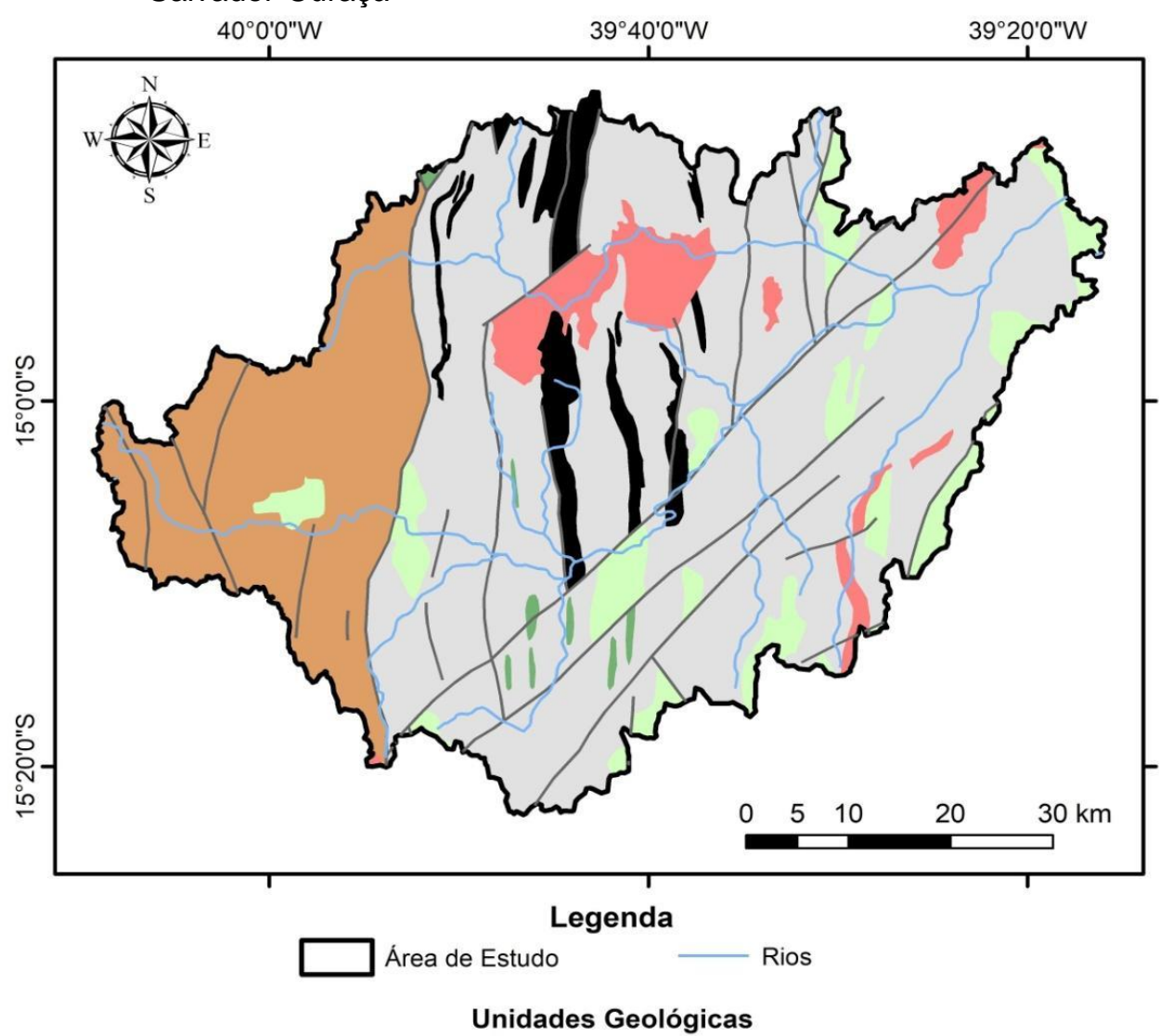

Complexo Ibicuí-Ipiaú

Rochas Supracrustais e Granitos Básicos

Fonte: Modificado de Pinho (2005). 
Segundo IBGE (1995), predomina na bacia o domínio geomorfológico da depressão Itabuna-Itapetinga, estendendo-se de SSW para NNE, margeado por serras e maciços pré-litorâneos. A depressão Itabuna-Itapetinga possui domínio pedológico de chernossolos e argissolos, respectivamente de oeste para leste, resultante das diferentes condições pluviométricas na bacia. Esses solos são pouco permeáveis, rasos e possuem altos teores de silte. Nas planícies de inundação do Rio Salgado, assim como em outros depósitos aluvionares, ocorre o domínio de planossolo (NACIF et al., 2003).

Segundo a classificação de Köppen (1936), o clima na BHRC varia de leste para oeste de acordo com a dinâmica das massas de ar, passando de clima tropical úmido (Af) a clima de monção (Am) e clima tropical com estação seca de Inverno (Aw), sofrendo interferências do Anticiclone Subtropical do Atlântico Sul (ENGELBRECHT et al., 2019; DEGOLA, 2013)

No final do século XIX, a região do semiárido baiano, que abrange os municípios de Itororó e Itapetinga na BHRC, passou por sucessivas secas, tornandose necessária a busca de alternativas para o armazenamento de água, sendo a mais comum delas a açudagem (REBOUÇAS, 1997). Essa técnica se espalhou por todo o território da seca, sendo estimado que aproximadamente 70 mil pequenos reservatórios tenham sido construídos até o final do século XX (MOLLE e CARDIER, 1992).

\subsubsection{Hidrologia}

A BHRC apresenta diminuição gradativa das taxas pluviométricas com o distanciamento da faixa litorânea (Figura 3), produzindo eventos de excessos hídricos na porção jusante da bacia em contraposição aos déficits hídricos nos municípios interioranos (ARCANJO et al., 1993). O período de maior incidência de chuvas ocorre entre os meses de novembro a abril. Os meses de janeiro e fevereiro são os mais quentes, enquanto julho e agosto os mais frios. A temperatura média anual oscila entre $20^{\circ} \mathrm{C}$ e $28^{\circ} \mathrm{C}$ (ENGELBRECHT et al., 2019 
Figura 3 - Distribuição da pluviometria na Bacia do Rio Cachoeira

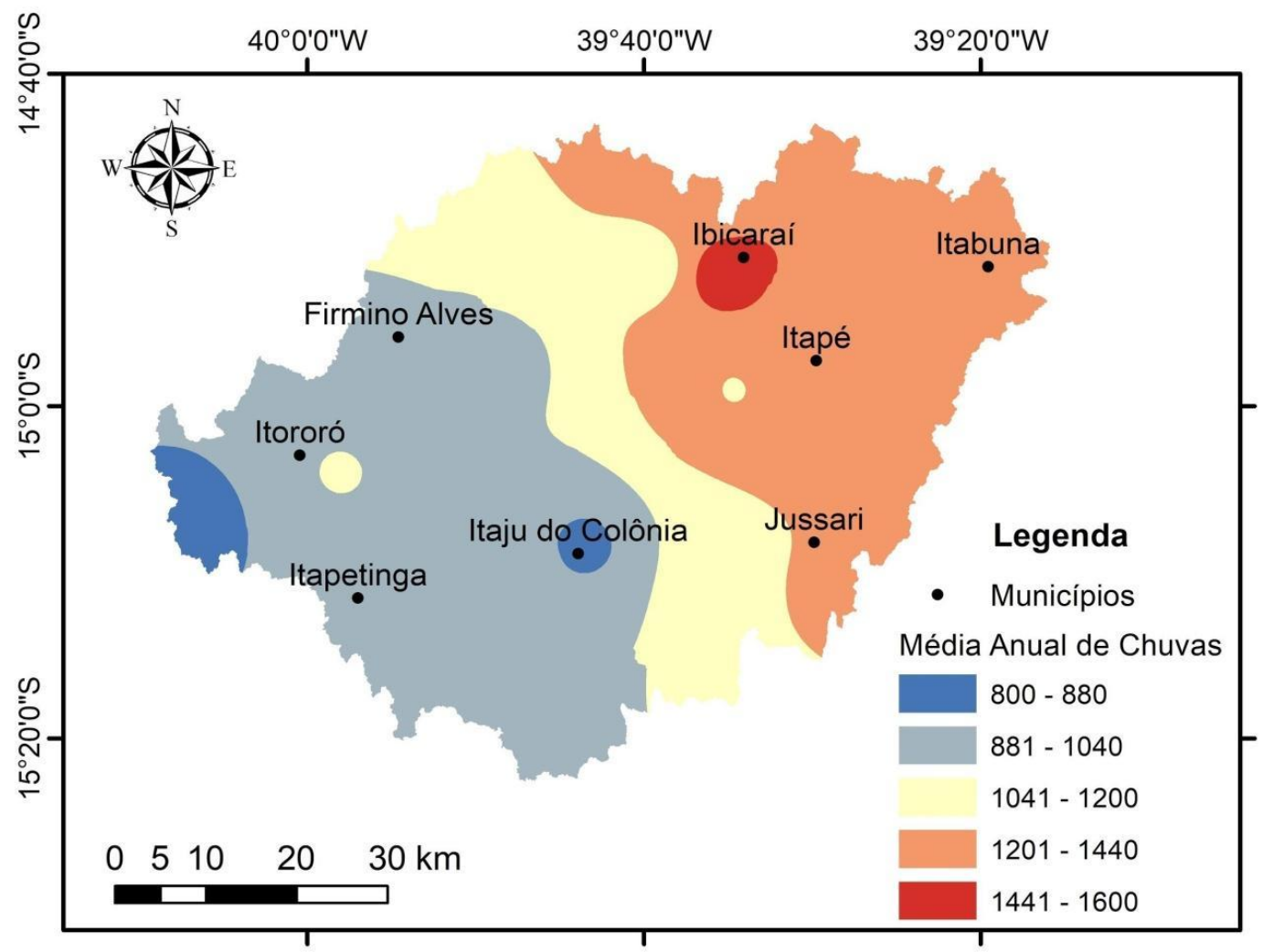

Fonte: Modificado de Engelbrecht et al. (2019).

O balanço hídrico da BHRC estima que $85 \%$ da precipitação retorna para a atmosfera na forma de evapotranspiração, enquanto cerca de $12 \%$ destina-se ao escoamento superficial, sugerindo baixa capacidade de infiltração no subsolo (ENGELBRECHT et al., 2019). As análises de hidroquímicas da interação água-rocha, realizadas por Teramoto et al. (2019), corroboram os resultados de Engelbrecht et al. (2019) e atestam a pequena taxa de recarga dos aquíferos.

\subsection{Sensoriamento Remoto}

Neste estudo foram utilizadas imagens multiespectrais obtidas pelo satélite Sentinel-2, que é equipado com sensor MSI (Multi Spectral Instrument) e fornece imagens com resoluções espaciais de $10 \mathrm{~m}, 20 \mathrm{~m}$ e $60 \mathrm{~m}$ (Tabela 1). A missão imageadora do Sentinel-2 é composta por dois satélites, Sentinel-2A e Sentinel-2B, de resolução temporal de 10 dias, com operação de um satélite, e de 5 dias, com operação dos dois satélites. 
Tabela 1 - Relação das bandas, comprimentos de onda e resolução espacial do satélite Sentinel-2

\begin{tabular}{ccc}
\hline Banda & $\begin{array}{c}\text { Comprimento de onda } \\
\text { central }(\mathbf{n m})\end{array}$ & $\begin{array}{c}\text { Resolução } \\
\text { Espacial (m) }\end{array}$ \\
\hline B1 - Aerossol & 443 & 60 \\
B2 - Azul & 490 & 10 \\
B3 - Verde & 560 & 10 \\
B4 - Vermelho & 665 & 10 \\
B5 - Red Edge 1 & 705 & 20 \\
B6 - Red Edge 2 & 740 & 20 \\
B7 - Red Edge 3 & 783 & 20 \\
B8A - Red Edge 4 & 865 & 20 \\
B8 - NIR & 842 & 10 \\
B9 - Water Vapor & 940 & 60 \\
B10 - Cirrus & 1375 & 60 \\
B11 - SWIR 1 & 1610 & 20 \\
B12 - SWIR 2 & 2190 & 20 \\
\hline
\end{tabular}

\subsubsection{Seleção e aquisição de imagens}

As imagens do satélite Sentinel-2 foram obtidas no website do Serviço Geológico dos Estados Unidos (https://earthexplorer.usgs.gov/). No período analisado, entre janeiro de 2015 e janeiro de 2019, foram selecionadas duas cenas, referentes a 11 de fevereiro de 2016 e a 04 de agosto de 2017.

A presença de nuvens na área de estudo, que produz sombras e impede a detecção de corpos d'água, foi fator limitante na seleção das imagens. Apesar do longo período analisado e da grande disponibilidade de imagens da região, apenas duas cenas apresentaram condições satisfatórias para o cálculo dos índices normalizados de água.

\subsubsection{Correção atmosférica}

Após a aquisição das imagens multiespectrais, foi realizado o préprocessamento de dados através da correção atmosférica, cujo objetivo foi transformar os valores de reflectância do topo da atmosfera (TOA - Top of Atmosphere) para reflectância da superfície terrestre (BOA -Bottom Of Atmosphere). 
As correções atmosféricas foram realizadas através do aplicativo Sen2Cor, do software SNAP 5.0, desenvolvido pela Agência Espacial Europeia (ESA) para correção atmosférica de imagens obtidas pelo Sentinel-2. O Sen2Cor obtém as informações de profundidade óptica do aerossol e do vapor d'água, utilizando as bandas 8A (865 $\mu \mathrm{m})$ e $9(945 \mu \mathrm{m})$, para o processo de correção atmosférica baseado em Look-upTables (ESA, 2010).

Durante as correções atmosféricas, as bandas utilizadas no cálculo do MNDWI foram reamostradas para resolução espacial de $20 \mathrm{~m}$. O software utilizado é disponibilizado gratuitamente pelo seguinte endereço eletrônico http://step.esa.int/main/third-party-plugins-2/sen2cor/sen2cor_v2-8 pela ESA.

\subsubsection{Identificação de corpos d'água}

O processamento digital das imagens multiespectrais e a identificação automática de corpos d'água foram realizados aplicando as metodologias propostas por McFeeters (1996) e Xu (2006), através do cálculo dos índices NDWI (Normalized Difference Water Index) e MNDWI (Modified Normalized Difference Water Index), respectivamente.

O NDWI utiliza as bandas do verde e do infravermelho próximo, conforme equação 1, para realçar a baixa reflectância dos comprimentos de onda entre o visível e o infravermelho próximo, o que permite a identificação de corpos d'água (DU et al., 2016).

$N D W I=\frac{\rho G r e e n-\rho N I R}{\rho \text { Green }+\rho N I R}$

Em que, $\rho$ Green refere-se à reflectância na faixa verde e $\rho N I R$ refere-se à reflectância na faixa do infravermelho próximo.

No caso do satélite Sentinel-2, as faixas do verde e do infravermelho próximo equivalem às bandas B03 e B08, respectivamente, ambas com resolução espacial de 10 m (Tabela 1). Assim, em cada pixel, o NDWI foi calculado pela seguinte equação:

$$
N D W I_{10 m}=\frac{\rho 03-\rho 08}{\rho 03+\rho 08}
$$


A versão modificada do $N D W I$, o $M N D W I$, substitui a banda do infravermelho próximo pela banda do infravermelho de ondas curtas, realçando o contraste entre corpos d'água, solo exposto e áreas construídas (XU, 2006). O índice MNDWI é calculado pela equação 3 .

$M N D W I=\frac{\rho G r e e n-\rho S W I R}{\rho G r e e n+\rho S W I R}$

Em que, $\rho$ Green se refere ao valor da reflectância na faixa verde e $\rho$ SWIR representa o valor de reflectância na banda do infravermelho de ondas curtas.

No caso do satélite Sentinel-2, as bandas do verde e do infravermelho de ondas curtas se referem às bandas $\mathrm{B} 03$ e B11, respectivamente. Em razão das diferentes resoluções espaciais entre as bandas $\mathrm{B} 03$ e B11, a banda B03 foi reamostrada para resolução espacial de $20 \mathrm{~m}$, utilizando o algoritmo nearest neighbor da ferramenta resampling do software SNAP 5.0. Assim, o MNDWI, com as resoluções espaciais apropriadas, foi calculado pela seguinte equação:

$M N D W I_{20 m}=\frac{\rho 03-\rho 11}{\rho 03+\rho 11}$

\subsection{Quantificação da área das pequenas represas}

O cálculo dos índices $N D W I$ e $M N D W I$, empregando as equações 2 e 4, resultaram em rasters cujos valores variam de 1 a -1 , sendo que os valores positivos representam pixels preenchidos por água, enquanto os valores negativos representam pixels não preenchidos por água.Para a quantificação da área ocupada por corpos d'água, os rasters com valores calculados de NDWI e MNDWI foram transformados em shapefile de pontos. A delimitação dos corpos d'água deu-se pela supressãodos pontos com valores negativos e manutenção dos pontos com valores positivos.

Para exclusão da interferência de nuvens e cursos d'água, como rios e córregos, foram elaboradas imagens $R G B$ de cor verdadeira (Figura 4), o que permitiu a inspeção manual das imagens e exclusão de pontos que não se referem aos reservatórios de água. 
Figura 4 - Imagem de composição RGB dos dias 11/02/2016 (a) e 04/08/2017 (b)

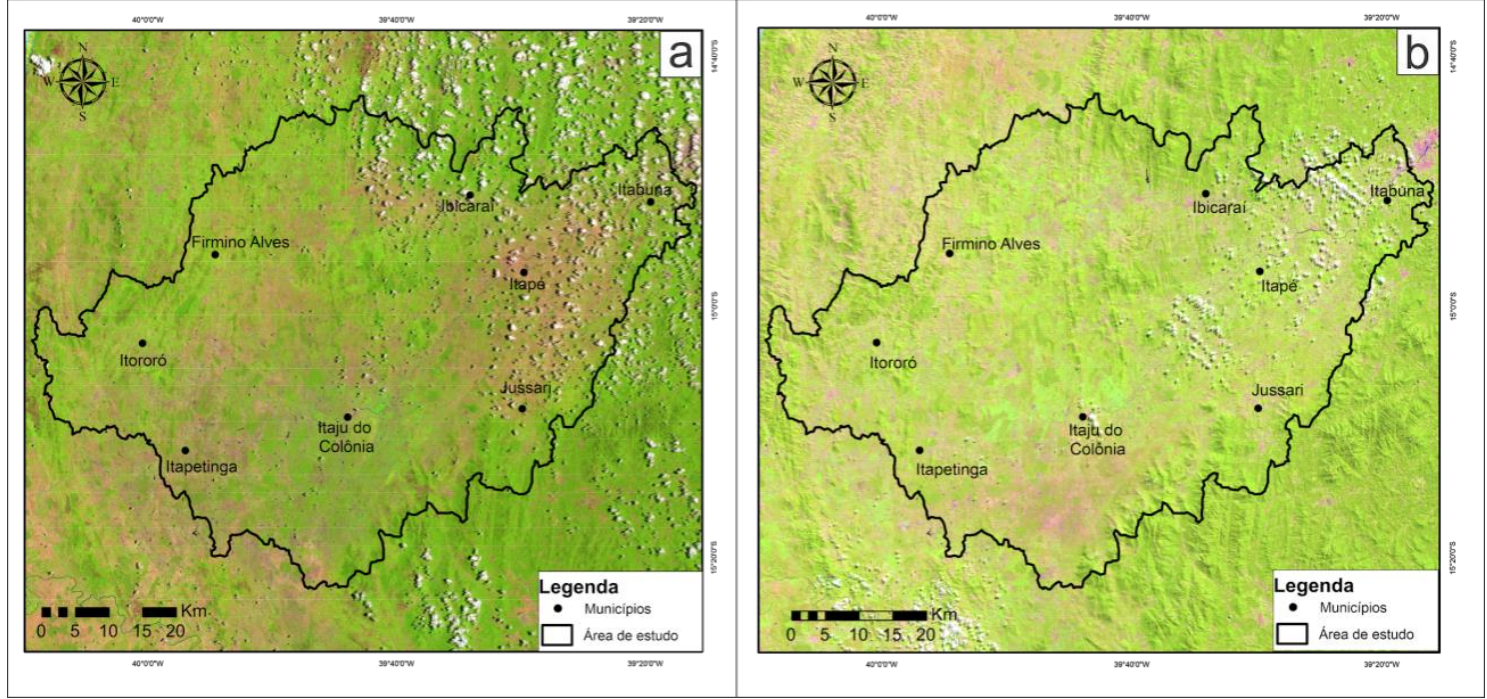

A quantificação da área ocupada pelos reservatórios de água foi efetuada pela ponderação entre a quantidade de pontos e a área do pixel, sendo $100 \mathrm{~m}^{2}$ para NDWI e $400 \mathrm{~m}^{2}$ para $M N D W I$. A área total ocupada pelos reservatórios de água foi determinada pela equação:

$A r=\sum n \cdot X m^{2}$

Onde $\Sigma n$ é o número de pontos dado como água e $X m^{2}$ representa a área do pixel, sendo $100 \mathrm{~m}^{2}$ para NDWI e $400 \mathrm{~m}^{2}$ para MNDWI.

\subsection{Densidade de represas ao longo da BHRC}

Para análise da distribuição dos reservatórios na BHRC, a área de estudo foi dividida em uma malha regular de $30 \mathrm{~km}^{2}$, compartimentando a bacia em 103 partes inteiras e 67 parciais. A densidade de reservatórios foi calculada pela proporção entre a área ocupada por reservatórios de água e a área total da BHRC na quadrícula (equação 6), resultando um índice adimensional que representa, em porcentagem, a área alagada em cada quadrícula.

$Q=\left(\frac{A r}{A}\right) \cdot 100$ 
Em que $Q$ é um índice adimensional referente a área alagada, expresso em porcentagem, Ar é a área dos corpos d'água presentes na quadrícula e A é a área da quadrícula.

\section{RESULTADOS}

\subsection{Identificação de corpos d'água na BHRC}

As diferentes metodologias empregadas para a identificação, delimitação e quantificação da área ocupada por pequenos reservatórios de água na BHRC apresentaram resultados consistentes (Tabela 3).

Na cena 1, o índice NDWI permitiu identificar 3,99 $\mathrm{Km}^{2}$ de área alagada na $\mathrm{BHRC}$, o que corresponde a $78 \%$ da área delimitada pelo índice $M N D W I$, que totalizou $5,09 \mathrm{Km}^{2}$. Na cena 2, o NDWI identificou cerca de 0,76 $\mathrm{Km}^{2}$ de área alagada, equivalente a $73 \%$ da área delimitada pelo $M N D W I$, que totalizou $1,04 \mathrm{Km}^{2}$ (Tabela 3). Os resultados mostram que a área alagada delimitada pelo índice NDWI foi sistematicamente inferior, entre $22 \%$ e $27 \%$, àquela delimitada pelo índice MNDWI.

Tabela 3 - Resultados da aplicação dos índices NDWI e MNDWI nas imagens do Sentinel-2

\begin{tabular}{|c|c|c|c|c|c|c|}
\hline Cena & Data & Índice & $\begin{array}{l}\text { No Pixels } \\
\text { de Água }\end{array}$ & $\begin{array}{c}\text { Área } \\
\text { Alagada } \\
\left(\mathbf{k m}^{2}\right)\end{array}$ & $\begin{array}{c}\text { Fração } \\
\text { Ocupada da } \\
\text { Bacia }\end{array}$ & $\begin{array}{l}\text { Proporção de } \\
\text { Área Alagada } \\
\text { (NDWI/MNDWI) }\end{array}$ \\
\hline \multirow[t]{2}{*}{1} & \multirow[t]{2}{*}{$11 / 02 / 2016$} & $N D W I(10 \mathrm{~m})$ & 39856 & 3,986 & $0,101 \%$ & \multirow[t]{2}{*}{$22 \%$} \\
\hline & & $M N D W I(20 \mathrm{~m})$ & 12725 & 5,090 & $0,129 \%$ & \\
\hline \multirow{2}{*}{2} & \multirow{2}{*}{ 04/08/2017 } & NDWI (10 m) & 7591 & 0,759 & $0,019 \%$ & \multirow{2}{*}{$27 \%$} \\
\hline & & MNDWI (20 m) & 2596 & 1,038 & $0,026 \%$ & \\
\hline
\end{tabular}

Os resultados obtidos pelos diferentes métodos e períodos avaliados indicam que os reservatórios de água ocupam área inferior a $0,13 \%$ da área total da bacia, evidenciando, no contexto da BHRC, pouca relevância desses corpos d'água.

Ambos os métodos aplicados para identificação automática dos corpos d'água, NDWI e MNDWI, indicaram diminuição da área alagada entre fevereiro de 2016, cena 1, e agosto de 2017, cena 2. A redução da área alagada foi cerca de $79,61 \%$ para 0 índice $M N D W I$ e de $80,96 \%$ para o NDWI. A figura 5 ilustra, em quadrícula específica 
da BHRC, a diminuição da área ocupada por reservatórios entre os diferentes períodos analisados.

Figura 5 - Comparação da área coberta por água em 02/2016 e 08/2017

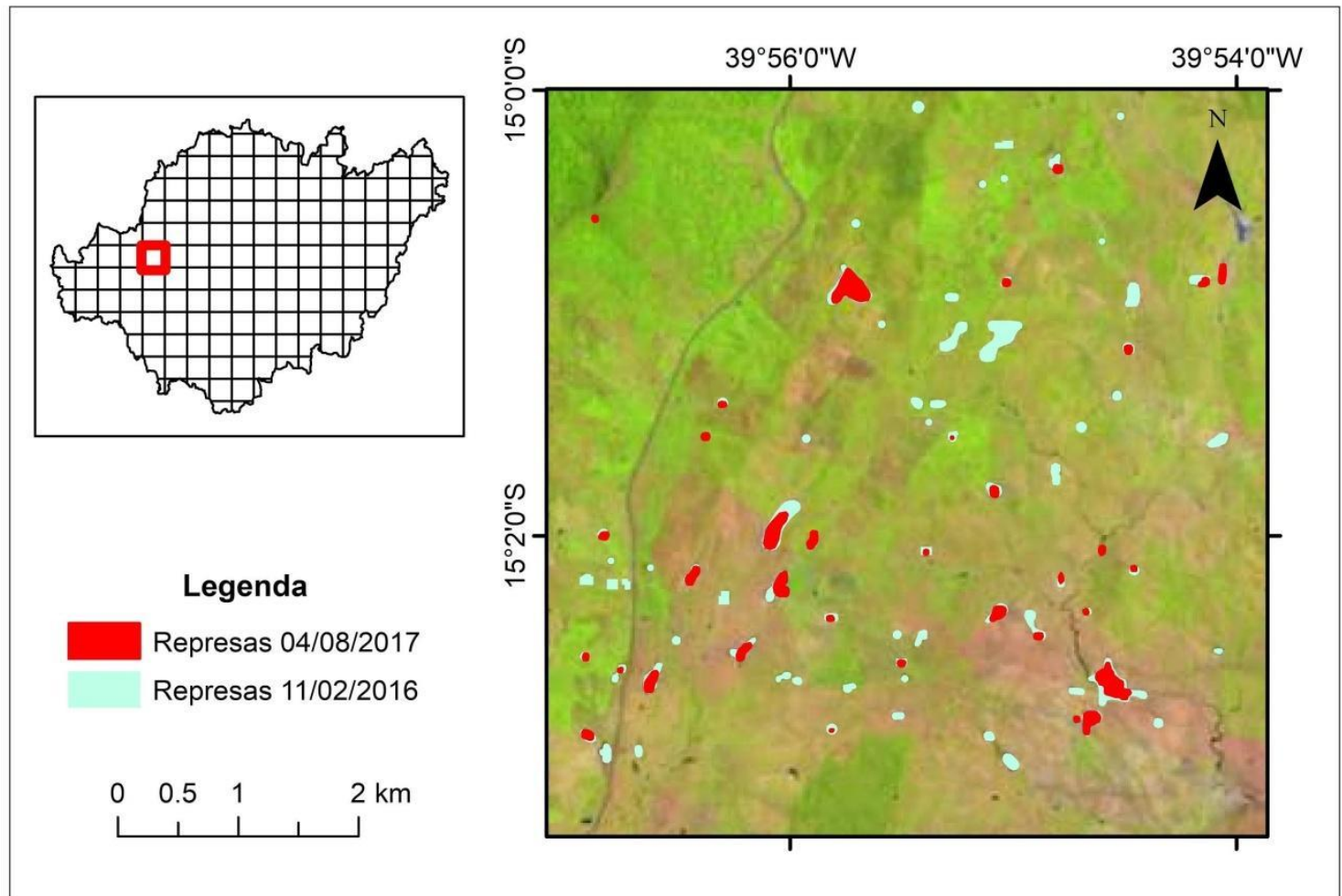

\subsection{Densidade de represas ao longo da BHRC}

A análise da concentração dos reservatórios de água na BHRC corrobora as variações sazonais observadas anteriormente e evidenciam a heterogeneidade espacial ao longo da BHRC (Figuras 6 e 7).

A quadrícula de destaque na porção norte da bacia, tanto no período de chuvas, quanto na estiagem, deve-se à Barragem Salomé, que representa extenso reservatório de água permanentedestinado ao abastecimento público e com uso complementar para a aquicultura.

No período chuvoso, cena 1, a região sudoeste da bacia, situada no polígono das secas, concentra os maiores índices de ocupação por reservatórios de água, perfazendo cerca de $0,86 \%$ a $0,6 \%$ da quadrícula, figura 6 a e 7 a, respectivamente. As demais regiões da bacia apresentam índices de concentração de reservatórios entre $0,0 \%$ e $0,4 \%$. Por outro lado, no período de estiagem, cena 2 , a distribuição dos reservatórios na $\mathrm{BHRC}$ torna-se mais homogênea e as áreas alagadas ocupam, no 
máximo, $0,5 \%$ da área da quadrícula, sendo que a grande maioria se encontra no intervalo entre $0,0 \%$ a $0,13 \%$.

Figura 6 - Percentual de área inundada, por quadrícula, na BHRC, detectado pelo índice MNDWI. a) Cena 1 de 11/02/2016 e b) Cena 2 de 04/08/2017

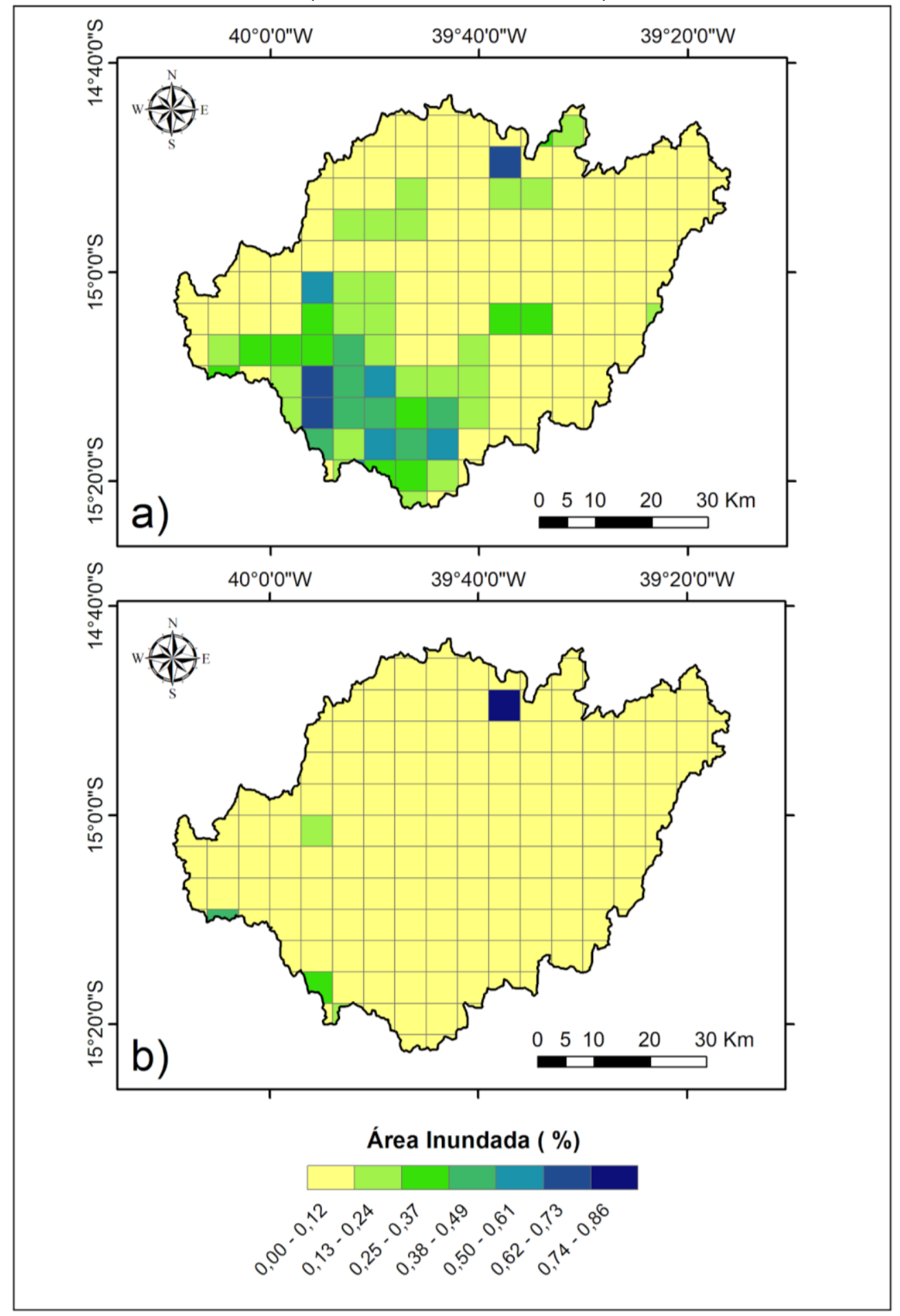


Figura 7 - Percentual de área inundada, por quadrícula, na BHRC, detectado pelo índice NDWI. a) Cena 1 de 11/02/2016 e b) Cena 2 de 04/08/2017

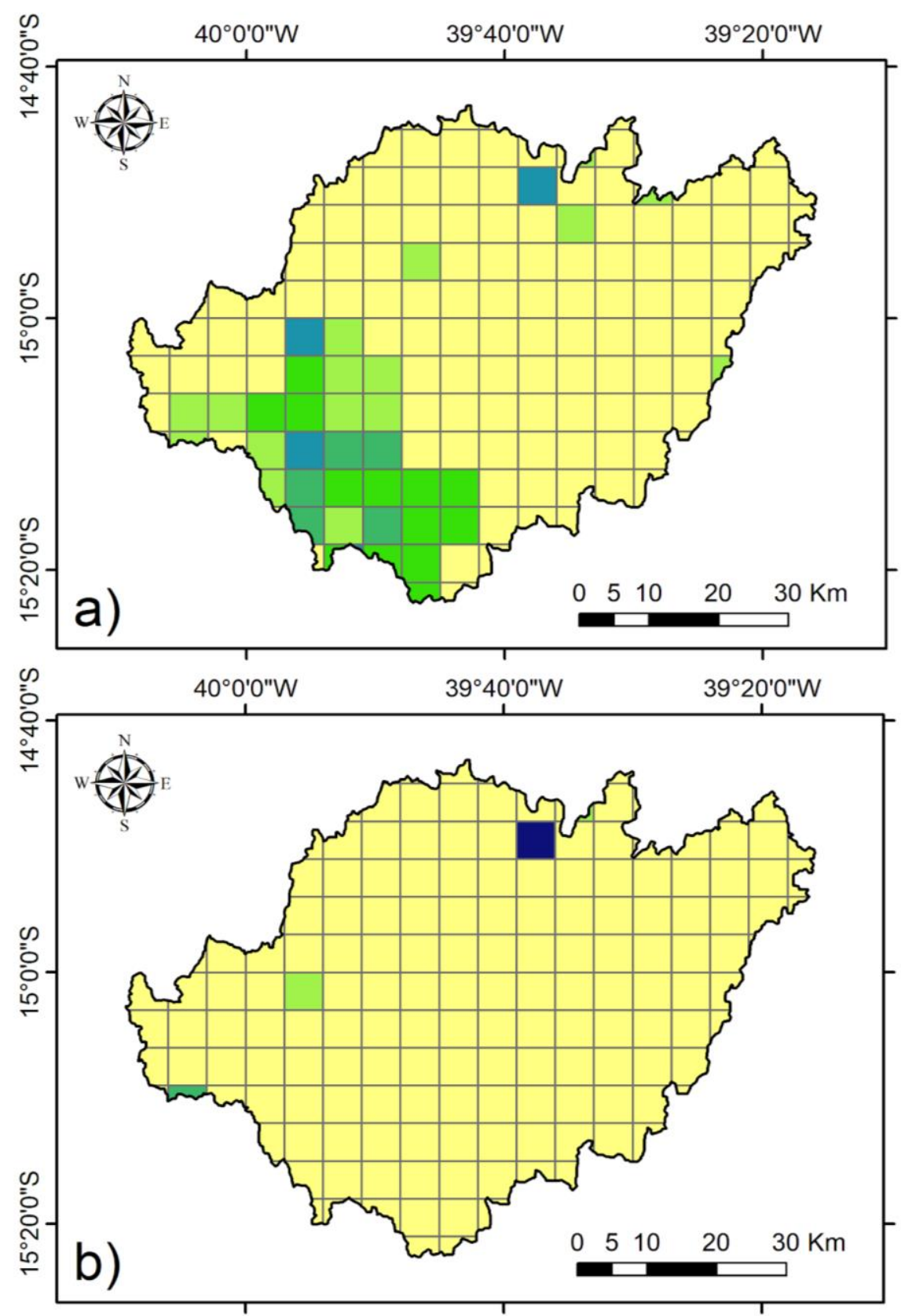

Área inundada (\%)

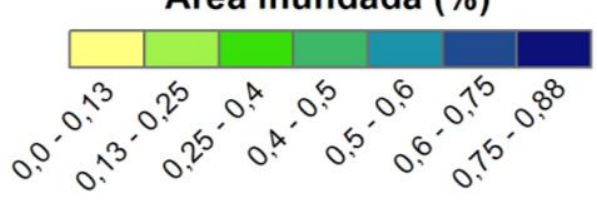




\section{DISCUSSÃO}

A utilização das imagens fornecidas pelo satélite Sentinel-2 foi definida devido sua boa resolução espacial e temporal. No entanto, apesar da adequação desses parâmetros, a constante presença de nuvens na BHRC foi fator limitante na seleção de imagens. Durante o período analisado, entre 2015 e 2019, foram selecionadas apenas duas cenas apropriadas para o processamento de dados. A limitação na disponibilidade de imagens adequadas impossibilitou a elaboração de uma série histórica da evolução dos pequenos reservatórios na BHRC, conforme propósito inicial deste trabalho.

A aplicação do método permitiu identificar e delimitar reservatórios de água na BHRC de maneira satisfatória. Ressalta-se que o índice NDWI, apesar de sua melhor resolução espacial, produziu resultados subestimados em relação ao índice $M N D W I$. Isso deve-se ao fato do índice MNDWI atribuir as áreas alagadas valores mais positivos, aumentando o contraste entre os alvos e permitindo distinguir com maior facilidade áreas alagadas de áreas construídas e de solo exposto. Apesar das diferenças absolutas obtidas entre os métodos MNDWI e NDWI, destaca-se que ambos índices registraram diminuição da área alagada em torno de $80 \%$ entre as cenas 1 e 2 .

A elevada concentração de reservatórios identificados na região sudoeste da BHRC (figura 6 e 7), sugere uma ação antrópica através da construção de pequenos reservatórios, açudes e barragens, para o armazenamento de água pluvial. As condições geológicas e climáticas dessa região, que abrange o escudo cristalino e o polígono das secas, não são favoráveis para formação de corpos d'água naturais. A aplicação da equação 6 , referente a densidade de represas, serviu como uma pertinente ferramenta para visualização dessa distribuição de corpos d'água na área.

Embora os resultados obtidos da quantificação de áreas alagadas demonstrem que os reservatórios, no contexto geral da bacia, apresentam pouca relevância e ocupam área inferior a $0,13 \%$, estes corroboram com os estudos de Engelbrecht et al. (2019) e Teramoto et. al. (2019), que estipularam uma evapotranspiração de $85 \%$ da água precipitada na BHRC, sem influência significativa de represas e reservatórios. 


\section{CONCLUSÕES}

Neste trabalho foram utilizadas imagens do satélite Sentinel-2 e aplicados os métodos NDWI e MNDWI para identificação automática de reservatórios de água na Bacia Hidrográfica do Rio Cachoeira (BHRC).

Os índices espectrais mostraram-se satisfatórios para identificação e delimitação de corpos d'água na área de estudo. No entanto, os resultados obtidos sugerem que a atuação singular da resolução espacial não substitui a aplicação de um índice mais eficiente, uma vez que o índice NDWI identificou, sistematicamente, menor proporção de áreas alagadas, quando comparados aos resultados obtidos pelo MNDWI.

Os resultados obtidos com os métodos empregados mostram que os reservatórios ocupam área insignificante da BHRC, inferior à $0,13 \%$ da área total, porém revelam a concentração desses corpos hídricos na região sudoeste da BHRC, região afetada pelo polígono das secas, e sugerem a ação antrópica através da construção de pequenos barramentos e açudes. Além disso, o estudo evidencia intensa variação sazonal na área, o que reduziu cerca de $80 \%$ de área inundada entre os períodos de fevereiro/2016 e agosto/2017, referentes à estação de verão e de inverno, respectivamente.

\section{REFERÊNCIAS}

ARCANJO, J. B. A. et al. Programa de levantamentos geológicos do básico do Brasil, Itabuna. Folha SD-24-YB-VI. Estado da Bahia. Escala 1: 100.000. Brasília: CPRM, 1993.

ARVOR, Damien et al. Monitoring thirty years of small water reservoirs proliferation in the southern Brazilian Amazon with Landsat time series. ISPRS journal of photogrammetry and remote sensing, v. 145, p. 225-237, 2018.

DOI:https://doi.org/10.1016/j.isprsjprs.2018.03.015

AVISSE, Nicolas et al. Monitoring small reservoirs' storage with satellite remote sensing in inaccessible areas. Hydrology and Earth System Sciences, v. 21, n. 12, p. 6445-6459, 2017. DOI: https://doi.org/10.5194/hess-21-6445-2017

BARBOSA, Johildo SF; SABATÉ, Pierre. Geological features and the Paleoproterozoic collision of four Archean crustal segments of the São Francisco Craton, Bahia, Brazil: a synthesis. Anais da Academia Brasileira de Ciências, v. 74, n. 2, p. 343-359, 2002. DOI: https://doi.org/10.1590/s0001-37652002000200009

CUNHA, Caroline Favoreto et al. Modelo área-volume para a Represa Guarapiranga empregando o índice NDWI. HolosEnvironment, v. 20, n. 1, 2020.

DOI: https://doi.org/10.14295/holos.v20i1.12370 
DEGOLA, T. S. D. Impactos e variabilidade do Anticiclone Subtropical do Atlântico Sul sobre o Brasil no clima presente e em cenários futuros. Instituto de Astronomia, Geofísica e Ciências Atmosféricas. Universidade de São Paulo, São Paulo, p. 91, 2013.

DU, Yun et al. Water bodies' mapping from Sentinel-2 imagery with modified normalized difference water index at 10-m spatial resolution produced by sharpening the SWIR band. Remote Sensing, v. 8, n. 4, p. 354, 2016.

DOI: https://doi.org/10.3390/rs8040354

ENGELBRECHT, Bruno Zanon et al. DISPONIBILIDADE HÍDRICA E BALANÇO HÍDRICO DA BACIA DO RIO CACHOEIRA NA REGIÃO DE ITABUNA/BA. Geosciences= Geociências, v. 38, n. 3, p. 731-740, 2019.

ESA - EUROPEAN SPACE AGENCY. GMES Sentinel-2 missionrequireddocument. 2010. Disponível em: http://esamultimedia.esa.int/docs/GMES/Sentinel-2 MRD.pdf

FRAZIER, Paul S. et al. Water body detection and delineation with Landsat TM data. Photogrammetric Engineering and Remote Sensing, v. 66, n. 12, p. 1461-1468, 2000.

IBGE - INSTITUTO BRASILEIRO DE GEOGRAFIA E ESTATÍSTICA. Manual técnico de geomorfologia. Rio de Janeiro: IBGE, n. 5, 1995.

KAPLAN, Gordana; AVDAN, Ugur. Object-based water body extraction model using Sentinel-2 satellite imagery. European Journal of Remote Sensing, v. 50, n. 1, p. 137-143, 2017. DOI: https://doi.org/10.1080/22797254.2017.1297540

LIEBE, J.; VAN DE GIESEN, N.; ANDREINI, Marc. Estimation of small reservoir storage capacities in a semi-arid environment: A case study in the Upper East Region of Ghana. Physics and Chemistry of the Earth, Parts A/B/C, v. 30, n. 6-7, p. 448-454, 2005. DOI: https://doi.org/10.1016/i.pce.2005.06.011

MCFEETERS, Stuart K. The use of the Normalized Difference Water Index (NDWI) in the delineation of open water features. International journal of remote sensing, v. 17, n. 7, p. 1425-1432, 1996. DOI: https://doi.org/10.1080/01431169608948714

MOLLE, F.; CADIER, E. Manual do pequeno açude. Recife: Sudene, 1992.

MUSTER, Sina et al. Water body distributions across scales: a remote sensing based comparison of three arctic tundra wetlands. Remote Sensing, v. 5, n. 4, p. 1498-1523, 2013. DOI: https://doi.org/10.3390/rs5041498

NACIF, P. G. S. et al. Ambientes naturais da bacia hidrográfica do rio Cachoeira. Cruz das Almas, Brasil, 2003.

OGILVIE, Andrew et al. Assessing floods and droughts in ungauged small reservoirs with long-term Landsat imagery. Geosciences, v. 6, n. 4, p. 42, 2016.

DOI: https://doi.org/10.3390/geosciences6040042

OGILVIE, Andrew et al. Surface water monitoring in small water bodies: potential and limits of multi-sensor Landsat time series. Hydrology and Earth System Sciences, v. 22, n. 8, p. 4349, 2018. DOI: https://doi.org/10.5194/hess-22-4349-2018

OGILVIE, Andrew et al. Socio-hydrological drivers of agricultural water use in small reservoirs. Agricultural Water Management, v. 218, p. 17-29, 2019.

DOI: https://doi.org/10.1016/j.agwat.2019.03.001 
PINHO, Ivana Conceição de Araújo. Geologia dos metatonalitos/metatrondhjemitos e granulitos básicos das regiões de Camamu-Ubaitaba-Itabuna, Bahia. 2005.

PÔSSA, ÉvelynMárcia; MAILLARD, Philippe. Precise delineation of small water bodies from sentinel-1 data using support vector machine classification. Canadian Journal of Remote

Sensing, v. 44, n. 3, p. 179-190, 2018. DOI: https://doi.org/10.1080/07038992.2018.1478723

REBOUÇAS, Aldo da C. Água na região Nordeste: desperdício e escassez. Estudos avançados, v. 11, n. 29, p. 127-154, 1997.DOI: https://doi.org/10.1590/s0103$\underline{40141997000100007}$

ROCHA FILHO, C. A. Diagnóstico Sócio-Econômico da Região Cacaueira, vol. 5: Recursos Hídricos. Comissão Executiva do Plano da Lavoura Cacaueira e Instituto Interamericano de Ciências Agrícolas-OEA. Rio de Janeiro, Cartográfica Cruzeiro do Sul. 92p, 1976.

TERAMOTO, Elias Hideo et al. Modeling rock-water interaction of fractured aquifer in itabuna/BA region. Anuario do Instituto de Geociencias, p. 735-741, 2019.

DOI: https://doi.org/10.11137/2019 1735741

WISSER, Dominik et al. The significance of local water resources captured in small reservoirs for crop production-A global-scale analysis. Journal of Hydrology, v. 384, n. 3-4, p. 264-275, 2010. DOI: https://doi.org/10.1016/j.jhydrol.2009.07.032

XU, Hanqiu. Modification of normalised difference water index (NDWI) to enhance open water features in remotely sensed imagery. International journal of remote sensing, v. 27, n. 14, p. 3025-3033, 2006. DOI: https://doi.org/10.1080/01431160600589179

YANG, Xiucheng et al. Mapping of urban surface water bodies from Sentinel-2 MSI imagery at $10 \mathrm{~m}$ resolution via NDWI-based image sharpening. Remote Sensing, v. 9, n. 6, p. 596, 2017. DOI: $\underline{\text { https://doi.org/10.3390/rs9060596 }}$

ZOU, Zhenhua et al. Continued decrease of open surface water body area in Oklahoma during 1984-2015. Science of the Total Environment, v. 595, p. 451-460, 2017.

DOI: https://doi.org/10.1016/j.scitotenv.2017.03.259 